\title{
JOURNAL OF LANGUAGE, LITERATURE AND TEACHING
}

(JLLTE)

Vol. 1, No. 1, April 2019, pp. 1-17

Available online at:

http://www.jllte.stbapia.ac.id

\section{浅谈印尼棉兰学习者学习普通话舌尖后辅音声母的情况}

\section{A Brief Talk on the Condition of How Learners in Medan Learn the Blade-palatal Initials in Standard Chinese}

\author{
李福祥 \\ Sichuan University, Chengdu, P.R. China
}

\section{摘 要}

舌尖后辅音声母一一就是尧舌音声母

$\mathrm{zh}[\mathrm{ts}] 、 \mathrm{ch}\left[\mathrm{ts} \mathrm{s}^{\mathrm{h}}\right] 、 \mathrm{sh}[\mathrm{s}] 、 \mathrm{r}[\mathrm{z}]$ 对于海外汉语学习者来说, 是学习汉语语音的最 大难点之一。在印尼, 不少有理想的学习者以发好尧舌音音节为汉语语音追 求目标, 因为这是普通话语音重要特征之一, 交际中也能防止别人误解意思 。本文内容是对印尼棉兰学习者普通话尧舌音声母的发音现象进行分析, 分 别华裔和非华裔学习者, 进行了普通话和闽南语声母以及普通话声母和印尼 语辅音的差异简介。本文目的是得出印尼, 尤其是棉兰学习者不能发准舌尖 后辅音声母的原因,

因此, 使用了定性研究法。对比结果为印尼语和闽南语没有舌尖后发音, 导致会说普通话的棉兰华人经常把尧舌音忽略掉，如“吃”应该发[tsh ${ }^{55}$ ], 他们发成 $\left[\mathrm{s}^{\mathrm{h}} \mathrm{\partial}\right.$ ?]。讲印尼语的当地人也经常把印尼语的这些辅音来代替尧舌 音, 即 [ t o ] 、 [ t s o ] 、 [ s o ] 、 [ r o ], 则学者 还按照汉语 拼音字母来念。

关键词印尼; 普通话语音; 舌尖后辅音声母 
JOURNAL OF LANGUAGE, LITERATURE AND TEACHING

(JLLTE)

Vol. 1, No. 1, April 2019, pp. 1-17

Available online at:

http://www.jllte.stbapia.ac.id

\title{
A Brief Talk on the Condition of How Learners in Medan Learn the Blade-palatal Initials in Standard Chinese
}

Antony Hardi

Sichuan University, Chengdu, P.R. China

\begin{abstract}
Blade-palatal initials zh[ts], $\operatorname{ch}\left[\mathrm{ts}{ }^{\mathrm{h}}\right.$ ], sh[s], and r[z] are important characteristics in Standard Chinese (Putonghua) pronunciation, which are also some difficult parts for non-Chinese speakers. This paper is intended to analyze the appearance and condition of the blade-palatal-initial pronunciation of learners in Medan, Indonesia, in which the learners are divided into two groups, Chinese Indonesians and locals. It also describes the brief differences between Standard Mandarin and Southern Min initials and between Standard Mandarin initials and Indonesian consonants. To achieve the objective, the research was conducted by using descriptive-qualitative design. Because there are no blade-palatal sounds either in Indonesian or in Southern Min, it is very common for Chinese descendants in Medan who speak Putonghua to always omit the blade-palatal parts, e.g.: "to eat" that should be pronounced as [ts ${ }^{\mathrm{h}}{ }^{55}$ ] to always be pronounced as [t $\mathrm{t}^{\mathrm{h}} \mathrm{\partial}$ ?], which is not correct and might lead to another meaning. As well as locals who speak Indonesian to always replace the blade-palatal sounds with the consonants which sound near to them: [tso], [ts ${ }^{\mathrm{h}}$ ], [sə], [rə].
\end{abstract}

Keywords: Indonesia; Standard Mandarin Phonetics; Blade-palatal Initials

\section{一、选题缘由}

印尼 2010 年总人口达到 2.38 亿人，其中有华裔 $2,832,510$ 人，占全国总人口 $1.20 \%$ 。根据研究, 印尼华人多数来自于中国的南方省份, 如福建、广东及海南。 因此, 印华大多数家里讲闽南语（包括漳泉话、潮汕话）、粤语（包括广州话、台 山话 ） 以 及 客家话等过 印尼是个多民族国家。根据 2010 年印尼统计中心机构（Badan Pusat

Statistik）的统计，印尼总共有 1,340

个民族。各个民族也有自己的语言, 但是在日常生活中他们都讲印度尼 


\section{JOURNAL OF LANGUAGE, LITERATURE AND TEACHING}

(JLLTE)

Vol. 1, No. 1, April 2019, pp. 1-17

西 亚 语 - - 印 尼 的 官 方 语 言 。舌尖后辅音声母也就是趐舌音声母

$\mathrm{zh}[\mathrm{ts}] 、 \mathrm{ch}\left[\mathrm{ts} \mathrm{h}^{\mathrm{h}}\right] 、 \mathrm{sh}[\mathrm{s}] 、 \mathrm{r}[\mathrm{z}]$ 是普通话语音重要特征之一, 对于海外汉语学习者来说 , 却是学习汉语语音的最大难点所在。 


\section{JOURNAL OF LANGUAGE, LITERATURE AND TEACHING}

(JLLTE)

Vol. 1, No. 1, April 2019, pp. 1-17

在印尼, 不少有理想的学习者以发好翅舌音为汉语语音追求目标—一发好趐舌音能 让汉语水平显得更有水准，交际中也不会让别人误解意思。印尼老华族人士很希望 改变他们的口音; 印尼不少学校聘请了中国老师, 其中重要的原因之一就是希望他 们能教会印尼学生标准的发音。 因此, 怎样克服困难, 学好教好普通话尧舌音声母, 成为新一代本土汉语教师 的富 有 挑战 性的课题。

\section{二、理论方法}

\section{（一） 中介语理论}

中介语最早由美国语言学家塞林格（Larry

Selinker）（1972），是试图探索第二语言习得者在习得过程中的语言系统和习得规 律的假说, 在第二语言习得的研究史上有重大意义。本文以此作为研究的理论依据

该理论认为, 学习者的语言既不同于目的语, 也不同于母语, 是一个独立的系 统，称为 “中介语”。刘珣教授总结了中介语的几个特点 ${ }^{1}$ :

1. 中介语在其发展过程中的任何一个阶段都是学习者创造的一种介于第一语 言和目的语之间的语言系统 ;

2. 中介语不是故意不变的, 而是一个不断变化的动态语系统；

3. 塞林克把中介语的产生原因归纳为：语言迁移、目的语规则的过度概括、 训练造成的迁移、学习者的学习策略和交集策略等五个方面；

4. 中介语的偏误有反复性 ;

5. 中介语的偏误有顽固性。

\section{（二）普通话尧羽舌音语音原理}

汉语语言学界对汉语声母 $\mathrm{zh} 、 \mathrm{ch} 、 \mathrm{sh}$ 的分析开始于 20 世纪 20 年代左右, 尤其是汉语和西方语言开始接触之后。早期的研究认为这三个音是“卷舌音 （retroflex）”, 但是关于发音位置，不同的学者有不同的看法：钱玄同（1920）认为 


\section{JOURNAL OF LANGUAGE, LITERATURE AND TEACHING}

(JLLTE)

Vol. 1, No. 1, April 2019, pp. 1-17

zh、ch、sh

这三个声母是是舌叶和硬腭阻, 并将北京话的这三个声母完全等同于英语中的舌叶音

${ }^{1}$ 刘珣. 对外汉语教育学引论[M]. 北京 : 语言文化大学出版社, $2000:$ P.169-P.170. 


\section{JOURNAL OF LANGUAGE, LITERATURE AND TEACHING \\ (JLLTE) \\ Vol. 1, No. 1, April 2019, pp. 1-17}

Available online at:

http://www.jllte.stbapia.ac.id

$[\mathrm{f}] 、\left[\mathrm{f}^{\mathrm{h}}\right] 、[\mathrm{~J}]$ 。而高本汉 (1994) 认为北京话中的 $\mathrm{zh}$ 和 ch 为舌尖前硬腭塞擦音,

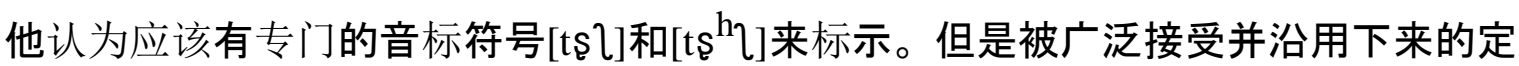
义是认为汉语声母 $\mathrm{zh}$ 、ch、sh 为“卷舌音”（Chao，1948，1968）。Chao 将其描述 为“舌尖前齿龈后音（apical post-alveolar）”，用国际音标中卷舌音符号 $[\mathrm{t} \S] 、\left[\mathrm{ts}^{\mathrm{h}}\right]$ 、 [s]来表示。赵元任所说的“舌尖前（apical）” 指的是主动发音器官为舌尖。发音时， 舌尖抬高并向后卷起, 使舌尖下表面接触或接近上齿龈后部。这一定义为诸多汉语

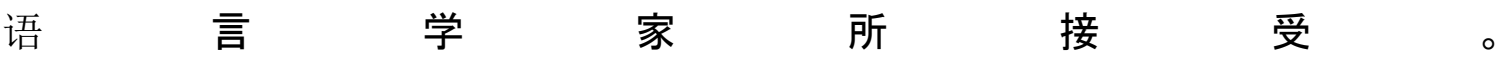

本文采用的是赵元任先生的原理以及定性和文献研究方法。

\section{三、 棉兰华裔学习者普通话翘舌音声母发音分析}

棉兰是印尼北苏门答腊省的省府, 是印尼的第三大城市, 仅次于雅加达和泗水。 棉兰的主要居民有原始马来族、马达族、美南加波族、亚齐族、华人、印度人 和欧洲人等。目前人口总数为 211 万，华人为 42.2 万人，大约占居民的 $20 \%$ 。

棉兰华人经常自称为唐人, 多数祖籍是中国福建省, 以闽南方言为第一语言。 虽然少数人在家里还会讲曾方言、潮州话、客家话等汉语方言, 但是所有棉兰华裔 都会说闽南语, 其为棉兰华裔的通用语。

\section{（一）闽南语和普通话语音差异简介}

闽南语是汉语闽方言的次方言, 该语言主要使用于中国福建南部、广东东部、海 南、台湾等; 海外主要集中在东南亚地区。中国大陆的语言学者多认为闽南语是一种 汉语方言, 西方学者则多认为是一种语言, 而中国台湾学者则两种说法皆有认

同者。 ${ }^{3}$ 本文认为闽南语是汉语的一种方言。

棉兰闽南语（简称为棉闽）是棉兰华人说的一种闽南语。其为居住于棉兰与周围 城市的华人的通用语。其发音和词汇接近福建漳州话的发音和词汇，还借鉴了一些印 尼语和英语里的词汇。陈丹风在 2000 年的《印尼的华人移民及其对印尼语的影响》中 共统计到闽南语词汇456个，而全部汉语借此大概511个包括饮食类、农作物 


\section{JOURNAL OF LANGUAGE, LITERATURE AND TEACHING}

(JLLTE)

Vol. 1, No. 1, April 2019, pp. 1-17

2 [印尼]《印广日报》，2008年2月3日.

3 蒋为文. 语言、文学 $k a p$ 台湾国家再想象 [M]. 台南 : 国立成功大学出版社, 2007. 
JOURNAL OF LANGUAGE, LITERATURE AND TEACHING

\section{(JLLTE)}

Vol. 1, No. 1, April 2019, pp. 1-17

Available online at:

$\underline{\text { http://www.jllte.stbapia.ac.id }}$

类、医药类、服布类、商业类、游戏娱乐类、航运类、节日类、宗教迷信类、社交 称呼类、建筑场所类以及其他中国特有的名物类。

中国福建的闽南语和棉兰的闽南语的发音有主要共同特征:

1. 念知、登等, 有时保留破裂音, 成 $[\mathrm{t}]$;

2. 无轻唇音 $[\mathrm{v}] 、[\mathrm{f}]$;

3. 有韵尾 $[-\mathrm{m}] 、[-\mathrm{p}] 、[-\mathrm{t}] 、[-\mathrm{k}]$;

4. 声调七类，与古代声调系统不尽相当。

表1 闽南语声母

\begin{tabular}{|c|c|c|c|c|c|c|c|c|c|c|c|}
\hline \multicolumn{3}{|c|}{$\begin{array}{l}\text { 发音部位 } \\
\downarrow \text { 发音方法 }\end{array}$} & $\begin{array}{l}\text { 双 } \\
\text { 唇 } \\
\text { 音 }\end{array}$ & $\begin{array}{l}\text { 唇 } \\
\text { 齿 } \\
\text { 音 }\end{array}$ & $\begin{array}{l}\text { 齿 } \\
\text { 间 } \\
\text { 音 }\end{array}$ & $\begin{array}{l}\text { 舌 } \\
\text { 尖 } \\
\text { 前 } \\
\text { 音 }\end{array}$ & $\begin{array}{l}\text { 舌 } \\
\text { 尖 } \\
\text { 中 } \\
\text { 音 }\end{array}$ & $\begin{array}{l}\text { 舌 } \\
\text { 尖 } \\
\text { 后 } \\
\text { 音 }\end{array}$ & $\begin{array}{l}\frac{\text { 舌 }}{\text { 面 }} \\
\text { 音 }\end{array}$ & $\begin{array}{l}\text { 舌 } \\
\text { 根 } \\
\text { 音 }\end{array}$ & $\begin{array}{l}\text { 喉 } \\
\text { 音 }\end{array}$ \\
\hline \multirow{3}{*}{ 塞音 } & \multirow[t]{2}{*}{ 清 } & $\begin{array}{l}\text { 不 } \\
\text { 送 } \\
\text { 气 }\end{array}$ & [p] & & & & {$[\mathrm{t}]$} & & & {$[\mathrm{k}]$} & \\
\hline & & $\begin{array}{l}\text { 送 } \\
\text { 气 }\end{array}$ & {$\left[\mathrm{p}^{\mathrm{h}}\right]$} & & & & {$\left[\mathrm{t}^{\mathrm{h}}\right]$} & & & {$\left[\mathrm{k}^{\mathrm{h}}\right]$} & \\
\hline & 浊 & & {$[\mathrm{b}]$} & & & & & & & {$[\mathrm{g}]$} & \\
\hline \multirow{3}{*}{$\begin{array}{c}\text { 塞擦 } \\
\text { 音 }\end{array}$} & \multirow[t]{2}{*}{ 清 } & $\begin{array}{l}\text { 不 } \\
\text { 送 } \\
\text { 气 }\end{array}$ & & & & [ts] & & & & & \\
\hline & & $\begin{array}{l}\text { 送 } \\
\text { 气 }\end{array}$ & & & & $\begin{array}{l}{[\mathrm{ts}} \\
\mathrm{h}\end{array}$ & & & & & \\
\hline & 浊 & & & & & & & & & & \\
\hline 鼻音 & 浊 & & {$[\mathrm{m}]$} & & & & [n] & & & [y] & \\
\hline 边音 & 浊 & & & & & & [1] & & & & \\
\hline \multirow{2}{*}{ 擦音 } & 清 & & {$[\Phi]$} & & & {$[\mathrm{s}]$} & & & & & [h] \\
\hline & 浊 & & & & & & & & & & \\
\hline $\begin{array}{c}\text { 半元 } \\
\text { 音 }\end{array}$ & 浊 & & & & & & & & & & \\
\hline
\end{tabular}


JOURNAL OF LANGUAGE, LITERATURE AND TEACHING

(JLLTE)

Vol. 1, No. 1, April 2019, pp. 1-17

Available online at:

http://www.jllte.stbapia.ac.id

表2 汉语普通话声母

\begin{tabular}{|c|c|c|c|c|c|c|c|c|c|c|}
\hline \multicolumn{3}{|c|}{ 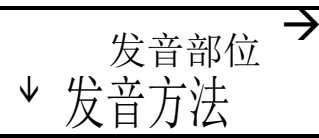 } & $\begin{array}{l}\text { 双唇 } \\
\text { 音 } \\
\end{array}$ & $\begin{array}{c}\text { 唇齿 } \\
\text { 音 } \\
\end{array}$ & $\begin{array}{c}\text { 齿间 } \\
\text { 音 } \\
\end{array}$ & $\begin{array}{l}\text { 舌尖 } \\
\text { 前音 }\end{array}$ & $\begin{array}{l}\text { 舌尖 } \\
\text { 中音 }\end{array}$ & $\begin{array}{l}\text { 舌尖 } \\
\text { 后音 } \\
\end{array}$ & $\begin{array}{c}\text { 舌面 } \\
\text { 音 } \\
\end{array}$ & $\begin{array}{c}\text { 舌根 } \\
\text { 音 } \\
\end{array}$ \\
\hline \multirow{3}{*}{ 塞音 } & 清 & $\begin{array}{l}\text { 不 } \\
\text { 送 } \\
\text { 气 }\end{array}$ & $\mathrm{b}[\mathrm{p}]$ & & & & $\mathrm{d}[\mathrm{t}]$ & & & $\mathrm{g}[\mathrm{k}]$ \\
\hline & & $\begin{array}{l}\text { 送 } \\
\text { 气 }\end{array}$ & $\mathrm{p}\left[\mathrm{p}^{\mathrm{h}}\right]$ & & & & $\mathrm{t}\left[\mathrm{t}^{\mathrm{h}}\right]$ & & & $\mathrm{k}\left[\mathrm{k}^{\mathrm{h}}\right]$ \\
\hline & 浊 & & & & & & & & & \\
\hline \multirow{3}{*}{ 塞擦音 } & 清 & $\begin{array}{l}\text { 不 } \\
\text { 送 } \\
\text { 气 }\end{array}$ & & & & $z[t \mathrm{t}]$ & & $\begin{array}{c}\mathrm{zh}[\mathrm{t} \\
\mathrm{s}]\end{array}$ & $\mathrm{j}[\mathrm{t} 6]$ & \\
\hline & & $\begin{array}{l}\text { 送 } \\
\text { 气 }\end{array}$ & & & & $\mathrm{c}\left[\mathrm{t}^{\mathrm{h}}\right]$ & & $\begin{array}{c}\mathrm{ch}[\mathrm{ts} \\
\mathrm{h}]\end{array}$ & $\mathrm{q}\left[\mathrm{t}^{\mathrm{h}}\right]$ & \\
\hline & 浊 & & & & & & & & & \\
\hline 鼻音 & 浊 & & $\mathrm{m}[\mathrm{m}]$ & & & & $\mathrm{n}[\mathrm{n}]$ & & & \\
\hline 边音 & 浊 & & & & & & $1[1]$ & & & \\
\hline \multirow{2}{*}{ 擦音 } & 清 & & & $\mathrm{f}[\mathrm{f}]$ & & $\mathrm{s}[\mathrm{s}]$ & & $\operatorname{sh}[\mathrm{s}]$ & $x[6]$ & $\mathrm{h}[\mathrm{x}]$ \\
\hline & 浊 & & & & & & & $\mathrm{r}[\mathrm{z}]$ & & \\
\hline 半元音 & 浊 & & & & & & & & & \\
\hline
\end{tabular}

从上表我们看出, 汉语普通话有舌尖后音 $\mathrm{zh}[\mathrm{ts}] 、 \operatorname{ch}\left[\mathrm{ts} \mathrm{s}^{\mathrm{h}}\right] 、 \mathrm{sh}[\mathrm{s}] 、 \mathrm{r}[\mathrm{z}]$, 但闽 南语没有。下面举例一些普通话尧舌音的字与闽南语（棉兰闽南语）念法对照： 
JOURNAL OF LANGUAGE, LITERATURE AND TEACHING

(JLLTE)

Vol. 1, No. 1, April 2019, pp. 1-17

Available online at:

http://www.jllte.stbapia.ac.id

表3 普通话尧羽舌音字与闽南语 (棉闽) 念法对照

\begin{tabular}{|c|c|c|c|}
\hline $\begin{array}{l}\text { 普通话尧 } \\
\text { 舌音声母 }\end{array}$ & 汉字 & 普通话念法 & 闽南语（棉闽）念法 \\
\hline \multirow{4}{*}{ zh } & 中 & zhōng[tşun $\left.{ }^{55}\right]$ & [t I On] \\
\hline & 猪 & zhū[tşu $\left.{ }^{55}\right]$ & {$[\mathrm{tu}]$} \\
\hline & 榨 & zhà[tşą ${ }^{51}$ ] & {$[\mathrm{ka}]$} \\
\hline & 真 & zhēn[tsən $\left.{ }^{55}\right]$ & [tsin] \\
\hline \multirow{4}{*}{ ch } & 长 & cháng[ts ${ }^{\mathrm{h}} \mathrm{an}^{35}$ ] & [təy] \\
\hline & 臭 & chòu[ts ${ }^{\mathrm{h}} \mathrm{ou}^{51}$ ] & {$\left[\mathrm{ts}^{\mathrm{h}} \mathrm{au}\right]$} \\
\hline & 冲 & chōng[tsh ${ }^{\text {un }}{ }^{55}$ ] & [th $\left.\mathrm{t}^{\mathrm{h}} \mathrm{ian}\right]$ \\
\hline & 茶 & chá $\left[t s^{h} A^{35}\right]$ & [tæ] \\
\hline \multirow{4}{*}{ sh } & 爽 & shuăng[suan $\left.{ }^{214}\right]$ & [s oฤ] \\
\hline & 水 & shuî[suei $\left.{ }^{214}\right]$ & [tsui]/[sui] \\
\hline & 师 & $\operatorname{sh} \overline{1}\left[s l^{55}\right]$ & {$[\mathrm{su}]$} \\
\hline & 神 & shén[ sən $\left.^{35}\right]$ & {$[\sin ]$} \\
\hline \multirow{4}{*}{$\mathrm{r}$} & 日 & $\operatorname{rì}\left[z \imath^{51}\right]$ & [dzit] \\
\hline & 热 & rè $\left[z \gamma^{51}\right]$ & [dzua?] \\
\hline & 荣 & róng[zuy ${ }^{35}$ ] & [en] \\
\hline & 入 & rù $\left[z u^{51}\right]$ & [dzip] \\
\hline
\end{tabular}

上表列出了一些普通话尧舌音的字, 但是可见闽南语都不念尧舌。上表的闽南语念 法是按照棉兰华人说的闽南语发音。虽然其基本上跟漳州话还是很相似的, 但是 已

经经过了演变, 包括声调和受印尼语影响的 [ d 3 ] 发音。所以 , 棉兰华裔学习者在学习尧舌音时感到十分困难。

（二）棉兰华裔学习者发普通话尧先音声母的难点 


\section{JOURNAL OF LANGUAGE, LITERATURE AND TEACHING}

(JLLTE)

Vol. 1, No. 1, April 2019, pp. 1-17

Available online at:

http://www.jllte.stbapia.ac.id

之所以棉兰华裔学习者学习普通话尧舌音声母 $\mathrm{zh}[\mathrm{ts}] 、 \operatorname{ch}\left[t \mathrm{~s}^{\mathrm{h}}\right] 、 \operatorname{sh}[\mathrm{s}] 、 \mathrm{r}[\mathrm{z}]$ 时感 到非常困难, 是因为他们的第一语言一一闽南语并没有其音。他们的平尧舌不分, 会说普通话的老一辈的华人也有平翘舌不分的现象。例如：

“我们当老师 $\left(\left[\mathrm{sa}^{44}\right]\right)$ 的一定要有责 ( [ to $\left.\left.{ }^{53}\right]\right)$ 任 $\left(\left[\mathrm{d} 32 \mathrm{n}^{53}\right]\right)$ 感去了解每个 学生 $\left(\left[\mathrm{s} \ni^{44}\right]\right)$ 。”

这句话除了元音和声调的不同以及入声问题之外，还有就是平尧舌音不分：“老 师”念成“老丝”, “学生”念成“学僧”。至于r该声母, 中年人一般会念 [d3], 而年轻人 因为受到印尼语的影响却会念 $[r]$ 。

四、棉兰非华裔学习者普通话尧羽舌音声母发音分析

除了华人, 棉兰非华裔的学习者主要有爪哇族（占33.03\%）和马达族（占20. 93\%）。4他们第一语言为爪哇语和马达语, 可是在日常生活中他们一般讲印尼语 。这些语言根本没有尧舌音。下图所示棉兰主要民族的比率 :

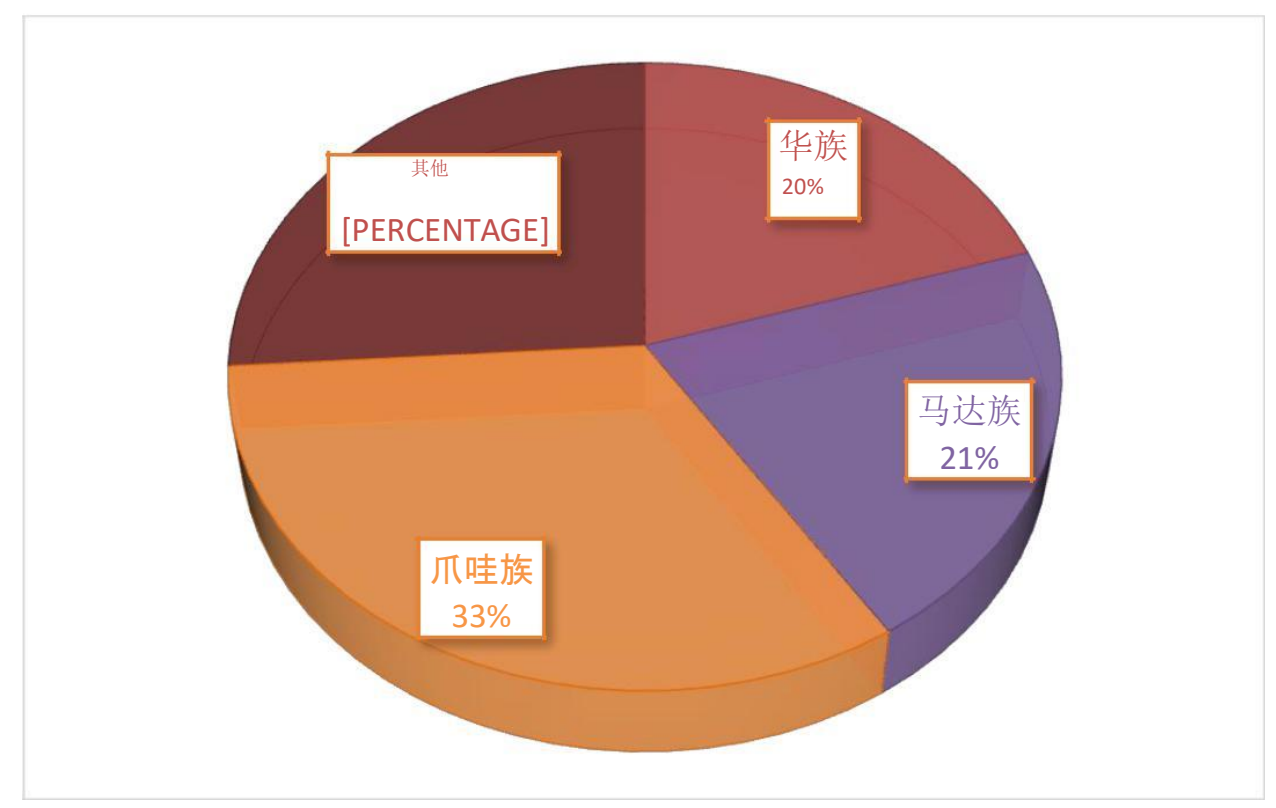

图1 印尼棉兰华裔 ( 华族) 与非华裔比率 


\section{JOURNAL OF LANGUAGE, LITERATURE AND TEACHING}

(JLLTE)

Vol. 1, No. 1, April 2019, pp. 1-17

${ }^{4}$ [印尼]北苏门答腊省统计中心机构Badan Pusat Statistik Sumatera Utara, 2000. 


\section{JOURNAL OF LANGUAGE, LITERATURE AND TEACHING}

\section{(JLLTE)}

Vol. 1, No. 1, April 2019, pp. 1-17

Available online at:

http://www.jllte.stbapia.ac.id

\section{（一） 印尼语和普通话语音差异简介}

\section{在语言学分类中，印度尼西亚语（Bahasa}

Indonesia）是以马来语为基础的印尼官方语言, 属于马来-

波利尼西亚语支，其底层源自于苏门答腊岛东北部，是一个地区性变种，同标准马 来语有超过 $80 \%$ 的同源性。印尼语具有 26 个字母辅音，其中 5 个元音和 19 个单辅音。 印尼语的辅音表如下（加上4个双辅音）：

印尼语辅音5

\begin{tabular}{|c|c|c|c|c|c|c|c|c|}
\hline \multicolumn{2}{|c|}{$\begin{array}{l}\text { 发音部位 }^{\rightarrow} \\
\downarrow \text { 发音方法 }\end{array}$} & \multirow{2}{*}{$\begin{array}{c}\text { 双唇音 } \\
\mathrm{p}[\mathrm{p}]\end{array}$} & \multirow[t]{2}{*}{ 唇齿音 } & $\begin{array}{l}\text { 舌尖 } \\
\text { 中音 } \\
\end{array}$ & 舌面音 & 舌根音 & $\begin{array}{l}\text { 混吅舍 } \\
\text { 叶音 } \\
\end{array}$ & 喉音 \\
\hline \multirow{2}{*}{ 塞音 } & 清 & & & $\mathrm{t}[\mathrm{t}]$ & & $\mathrm{k}[\mathrm{k}]$ & & \\
\hline & 浊 & $\mathrm{b}[\mathrm{b}]$ & & $\mathrm{d}[\mathrm{d}]$ & & $\mathrm{g}[\mathrm{g}]$ & & \\
\hline \multirow{2}{*}{ 塞擦音 } & 清 & & & & & & $\mathrm{c}[\mathrm{ts}]$ & \\
\hline & 浊 & & & & & & $\mathrm{j}[\mathrm{d} 3]$ & \\
\hline 鼻音 & & $\mathrm{m}[\mathrm{m}]$ & & $\mathrm{n}[\mathrm{n}]$ & ny[n] & $\operatorname{ng}[\mathrm{y}]$ & & \\
\hline 边音 & & & & $1[1]$ & & & & \\
\hline 嘼音 & & & & $\mathrm{r}[\mathrm{r}]$ & & & & \\
\hline \multirow{2}{*}{ 擦音 } & 清 & & $\mathrm{f}[\mathrm{f}]$ & $\mathrm{s}[\mathrm{s}]$ & & $\mathrm{kh}[\mathrm{x}]$ & $\operatorname{sy}\left[\int\right]$ & $\mathrm{h}[\mathrm{h}]$ \\
\hline & 浊 & & $\mathrm{v}[\mathrm{v}]$ & $\mathrm{z}[\mathrm{z}]$ & & & & \\
\hline 半元音 & & $\mathrm{w}[\mathrm{w}]$ & & & $y[j]$ & & & \\
\hline
\end{tabular}

说明：表中的混合舌叶音 c [ t ] 、 j [ d 3 ] 是 带有舌叶色彩的舌尖音。

从上表所列发音部位来看, $\left.\mathrm{z}[\mathrm{t}] 、 \mathrm{c}^{\mathrm{h}} \mathrm{t}^{\mathrm{h}}\right] 、 \mathrm{~s}[\mathrm{~s}]$ 在汉语普通话中是舌尖前音（见表

2），在印尼语的语音系统中

$z[z] 、 s[s]$ 是舌尖中音, $c[t]$ 是混吅舌叶音。汉语普通话有舌尖后音 $z h[t s] 、 c h[t s h$ ]、sh[s]、r[z]，但与闽南语一样，印尼语也没有。 


\section{JOURNAL OF LANGUAGE, LITERATURE AND TEACHING}

(JLLTE)

Vol. 1, No. 1, April 2019, pp. 1-17

因此, 棉兰的母语或第一语言为印尼语的非华裔学习者在学习这些音时也会遇 到同样的困难。

\footnotetext{
5 梁敏和主编. 印度尼西亚语三百句[M]. 北京 : 北京大学出版社, 1995.
} 


\section{JOURNAL OF LANGUAGE, LITERATURE AND TEACHING}

(JLLTE)

Vol. 1, No. 1, April 2019, pp. 1-17

Available online at:

http://www.jllte.stbapia.ac.id

\section{（二）棉兰非华裔学习者发普通话尧羽舌音声母的难点}

如上文所析, 华裔学习者和非华裔学习者因为印尼语没有尧舌音的缘故, 导致 了学习汉语普通话尧舌音时碰到较大困难。另一方面, 从文字上说, 印尼文和汉语 拼音两者用的都是拉丁字母, 所以除了平尧舌音不分的现象, 则学的印尼学习者 ( 尤其非华裔）习惯把汉语拼音的zh[ts]、ch[ts $\left.{ }^{\mathrm{h}}\right] 、 \mathrm{sh}[\mathrm{s}]$ 用印尼语的读法来念。例如

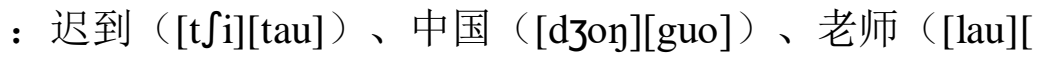

Si]）等, 同时, 印尼语是没有声调的。印尼非华裔则学习者学习汉语时, 声调也是 难点之一。

除了没有尧舌音, 印尼语也没有送气音。这导致讲印尼语的学生除了不会发翘 舌音, 特别对声母 $\mathrm{ch}\left[\mathrm{ts} \mathrm{s}^{\mathrm{h}}\right.$ 也有一定的困难。

印尼语还有一个特点, 就是有颤音 $r[r]$ 。一些较常用的带颤音词如：rasa （[rasa], 味道）、transportasi（[transportasi], 交通）、presiden（[presiden], 总 统) 等。因此, 印尼学生(尤其非华裔)经常把汉语普通话尧舌音声母r[z]念颤, 如: 人（[rən]）、荣耀（[ruy][jau]）、让（[ray]）等。

五、总结

从对比闽南语、印尼语和普通话的声母与辅音来看, 可以得出造成印尼人学习 者 (华裔、非华裔) 学习尧舌音声母困难的重要原因是什么, 重要原因就是因为学 生的第一语言——印尼语和闽南语均没有尧舌音, 所以普通话尧舌音成为他们学习 的 难 点

\section{六、参考文献}

[1] 白芸. 质的研究[M]. 北京: 教育科学出版社, 2009.

[2] Chao, Y. R. Mandarin Primer [M]. Cambridge, Mass.: Harvard University Press. 1948. 


\section{JOURNAL OF LANGUAGE, LITERATURE AND TEACHING}

(JLLTE)

Vol. 1, No. 1, April 2019, pp. 1-17

Available online at:

http://www.jllte.stbapia.ac.id

[3] Chao,Y. R. A Grammar of Spoken Chinese [M]. Berkeley: University of California Press. 1968.

[4] 陈丹凤. 印尼的华人移民及其对印尼语的影响[D]. 厦门大学学士学位论文, 2000.

[5] 陈瑶. 课堂观察指导[M]. 北京: 教育科学出版社, 2003.

[6] 董琳莉. 印尼华裔学生学习普通话语音的难点及其克服办法[J]. 汕头大学学报, 1997.

[7] 冯勇强, 则敏, 贺琳, 吕士楠. 汉语话语音节时长统计分析[M].

北京: 清华大学出版社, 2001.

[8] 傅碧达. 印度尼西亚高中汉语教学研究[D]. 东北师范大学硕士学位论文, 2010.

[9] 高名凯. 语言论[M]. 北京: 商务印书馆, 1995.

[10] 国家语言文字工作委员会普通话测试中心编制. 中华人民共和国教育部语言文 字应用管理司组织审定：普通话水平测试实施纲要 $[\mathrm{J}]$.北京：商务印书馆， 2004.

[11] 胡裕树主编. 现代汉语[M]. 上海：上海教育出版社， 1995.

[12] 黄伯荣, 廖序东. 现代汉语（上册）[M]. 北京：高等教育出版社， 2002.

[13] 季安锋, 蔡爱娟. 印尼学生习得汉语语音难点分析[J]. 海外华文教育, 2013.

[14] 蒋为文. 语言、文学kap台湾国家再想象 $[\mathrm{M}]$. 台南: 国立成功大学出版社, 2007.

[15] 孔远志. 印度尼西亚语发展史[M]. 北京：北京大学出版社， 1992.

[16] Krashen, D. Stephen. Principles and Practice in Second Language Acquisition [M]. Pergamon Press Inc. 1982.

[17] 李如龙. 现代汉语方言音库闽语概况库 $[M]$. 上海: 上海教育出版社, 2003.

[18] Li, Zhuqing. Minnan-English Dictionary [M]. Hyattsville, MD: Dunwoody Press. 2008.

[19] 梁敏和主编. 印度尼西亚语三百句[M]. 北京: 北京大学出版社, 1995.

[20] 刘润清, 胡壮麟主编. 外语教学中的科研方法[M].

北京: 外语教学与研究出版社, 1999.

[21] 刘珣. 对外汉语教育学引论[M]. 北京: 北京语言大学出版社, 2000.

[22] 刘珣. 汉语作为第二语言教学简论[M]. 北京: 北京语言大学出版社, 2007. 


\section{JOURNAL OF LANGUAGE, LITERATURE AND TEACHING}

(JLLTE)

Vol. 1, No. 1, April 2019, pp. 1-17

Available online at:

http://www.jllte.stbapia.ac.id

[23] 吕必松. 汉语和汉语作为第二语言教学[M]. 北京: 北京大学出版社, 2007.

[24] Rubin, D. \& Smith, K. Effects of Accent, Ethnicity, and Lecture Topic on Undergraduates' Perceptions of Nonnative English-speaking Teaching Assistants [J]. International Journal of Intercultural Relations. 1990.

[25] 吴中伟, 郭鹏. 对外汉语任务型教学[M]. 北京: 北京大学出版社, 2009.

[26] 肖祥忠. 难度等级与印尼汉语教学 [C].

云南师范大学学报（对外汉语教学与研究版）,2007.

[27] 谢小丽. 日本学习者汉语普通话尧舌音习得研究 [J]. 吆林工程技术师范学院学 报，2010.

[28] 杨碧珠. 关于普通话和闽方言平尧舌音的辨正[J]. 宁德师专学报, 1995.

[29] 张和生. 汉语可以这样教——语言要素篇[M]. 北京: 商务印书馆, 2006. 